\title{
Saúde sexual e reprodutiva: competências da equipe na Atenção Primária à Saúde
}

\author{
Sexual and reproductive health: \\ team competences in Primary Health Care services
}

Shana Vieira Telo ${ }^{1}$

Regina Rigatto Witt ${ }^{1}$
${ }^{1}$ Escola de Enfermagem, Universidade Federal do Rio Grande do Sul. R. São Manoel 963, Santa Cecília. 90620-110 Porto Alegre RS Brasil.

shanavt@hotmail.com

\begin{abstract}
Sexual and reproductive health rights were developed recently as a result from the movements held for Human Rights and citizenship. Delimitations of this subject have not been explored in Brazil yet, even though the importance of developing skills related to this subject is recognized. This paper aims to construct a Sexual and Reproductive health transversal skills framework based on specialists' point of view. A mix methods descriptive exploratory research with the use of the Delphi Technique was developed with 41 specialists in sexual and reproductive health and rights. Three rounds of data gathering were carried out. Of the 36 skills resulting from the qualitative analysis, 32 achieved a general consent and were classified in four domains: ethics and professional principles; leadership and management; community work, health and education, counseling and evaluation; and health care. Results corroborate skills content recommended by the international literature. These skills, which are transversal, may support the development of actions and practices of the health professionals concerning sexual and reproductive health care.

Key words Sexual and reproductive health, Sexual and reproductive rights, Professional competence, Primary health care
\end{abstract}

Resumo Os direitos à saúde sexual e reprodutiva foram desenvolvidos recentemente advindos da luta pela cidadania e pelos Direitos Humanos. Embora haja um reconhecimento da necessidade de formação por competências nesta área, sua delimitação ainda não foi explorada no Brasil. Este trabalho teve como objetivo construir um referencial de competências transversais para a atenção em Saúde Sexual e Reprodutiva na Atenção Primária à Saúde a partir da visão de especialistas. Trata-se de um estudo exploratório-descritivo, de abordagem mista, através da técnica Delphi online, realizado com 41 especialistas em saúde Sexual e reprodutiva e direitos sexuais e reprodutivos. Foram realizadas três rodadas de Delphi. Das 36 competências resultantes da análise qualitativa, 32 alcançaram consenso, sendo classificadas em quatro domínios: ética e princípios profissionais; liderança e gestão; trabalho com a comunidade, saúde e educação, aconselhamento e avaliação; $e$, provisão do cuidado. Os resultados corroboram conteúdos de competências preconizadas na literatura internacional e apresentam inovações para a prática. Por serem transversais, estas podem subsidiar o planejamento de ações das equipes no âmbito da saúde sexual e reprodutiva.

Palavras-chave Saúde sexual e reprodutiva, Direitos sexuais e reprodutivos, Competência profissional, Atenção Primária à Saúde 


\section{Introdução}

Os direitos à saúde sexual e reprodutiva (DSR) foram reconhecidos recentemente e são considerados uma conquista histórica, advinda da luta pela cidadania e pelos Direitos Humanos ${ }^{1}$. Abrangem o exercício da vivência da sexualidade sem constrangimento, da maternidade voluntária e da anticoncepção autodecidida ${ }^{2}$. Estes direitos devem ser percebidos para que sejam pensadas estratégias governamentais eficazes, uma vez que o atendimento das demandas por cuidado à saúde sexual e reprodutiva (SSR) relacionadas às vivencias de homens e mulheres deve ser um compromisso das políticas públicas na perspectiva da integralidade ${ }^{3}$.

As ações em saúde sexual e reprodutiva tem como marco legal a Conferência Internacional sobre População e Desenvolvimento (CIPD), que as definiu como primordiais à saúde, aos direitos sexuais e aos direitos reprodutivos, abandonando a ênfase na necessidade de limitar o crescimento populacional e a IV Conferência Mundial sobre a Mulher, que apresenta avanço na definição dos direitos sexuais e direitos reprodutivos como Direitos Humanos ${ }^{1,4}$.

No Brasil, a Política Nacional de Atenção Integral à Saúde da Mulher e, principalmente, a Política Nacional dos Direitos Sexuais e dos Direitos Reprodutivos orientam as ações em saúde sexual e reprodutiva ${ }^{1}$.

O conceito de direito reprodutivo passa a ser classificado como um ato político, ponderando conversas e negociações ${ }^{2}$, porém, essa mudança de enfoque, é complexa e demanda tempo, uma vez que implica em transformações culturais da sociedade e necessita estar focada na educação em saúde e no protagonismo dos atores envolvidos com o cuidado, através do diálogo, co-responsabilização e reflexão acerca dos mesmos ${ }^{5}$.

A utilização do termo planejamento reprodutivo em substituição a planejamento familiar tem embasado a política de SSR no Brasil, havendo a defesa de que se trata de uma concepção mais abrangente, tendo em vista que o conceito de planejamento familiar, assim como o de controle de natalidade, tem também um enfoque econômicodemográfico.

A consolidação dos direitos sexuais se deu apenas na década de 1990 e, na atualidade, uma parcela dos movimentos feministas utiliza esta terminologia na luta das desigualdades de gênero $^{6}$. A dimensão da sexualidade surge como um tema polêmico e como grande dificuldade para progresso, devido aos tabus e preconceitos que permeiam a discussão ${ }^{7}$.
O modo como as instituições e as práticas de saúde se organizam, reproduzem este contexto, oferecendo uma atenção fragmentada e de baixa resolutividade, com o cuidado dirigido a queixas clínicas e ainda muito direcionadas ao público feminino $^{5}$, cujos serviços ainda apresentam características predominantes curativas, com poucas ações de prevenção e promoção na Saúde Sexual e Reprodutiva ${ }^{8}$. O concreto exercício dos direitos sexuais e reprodutivos demanda políticas públicas que garantam a saúde sexual e reprodutiva e que tem na APS uma das suas grandes áreas de atuação. Portanto, a exigência de que o Estado assegure esses direitos está diretamente proferida aos processos de trabalho dos profissionais de saúde, de forma que, dependendo de sua postura no atendimento aos usuários (as), tal garantia pode ser comprometida.

Neste sentido, torna-se importante para propiciar o diálogo, entre os sujeitos envolvidos com o cuidado, de uma proposta educativa que não considere apenas o modelo biomédico, mas que pondere questões relativas ao gênero, à sexualidade, à autonomia e à liberdade para a construção de práticas não discriminatórias que garantam a promoção, a proteção e o exercício da sexualidade e da reprodução como um direito, pautadas na integralidade da atenção, como norteadoras das práticas assistenciais no âmbito da atenção à saúde².

Para um maior entendimento a respeito deste contexto, realizou-se um estudo abordando a temática do trabalho das equipes no cuidado a saúde sexual e reprodutiva tendo como objeto de pesquisa as competências transversais da equipe de saúde necessárias para a atenção em saúde sexual e reprodutiva na APS. Para tanto, tornou-se necessária a aproximação aos conceitos de competência, que vem sendo descritos na literatura como a capacidade de articular e mobilizar conhecimentos, habilidades e atitudes, colocando-os em ação para resolver problemas e enfrentar situações de imprevisibilidade em uma determinada situação de trabalho e contexto cultural ${ }^{9}$. Gebbie e Gill $^{10}$ definem as competências transversais como aquelas que transcendem os limites das disciplinas específicas e ajudam a unificar a prática no âmbito da saúde pública. Desta forma, pretende-se com este estudo proporcionar aos profissionais envolvidos com o cuidado uma reflexão sobre suas práticas na identificação e análise das competências que acreditam ser necessárias para realizar um cuidado de qualidade aos usuários.

Na Saúde Pública, as competências facilitam a comunicação entre as linhas programáticas e organizacionais, bem como fortalecem o cresci- 
mento profissional ${ }^{10}$. É importante pensarmos em um conjunto de competências para programas preconizados para a assistência como a SSR, para grupos de trabalhadores ou serviços essenciais, reforçando a importância do trabalho interdisciplinar no contexto da APS.

$\mathrm{Na}$ literatura internacional documentos e estudos sobre estas competências abordam a necessidade de formação de competências para atuação na área da sexualidade através da proposta de um programa de treinamento e avaliação clínica de saúde sexual e reprodutiva para profissionais de saúde da APS ${ }^{11}$; a necessidade de formação de enfermeiros para atuar na saúde sexual e reprodutiva $^{12}$; a avaliação do conhecimento sobre estas questões com médicos no Paquistão ${ }^{13}$; as barreiras e desafios encontrados pelos profissionais da APS para realizar o aconselhamento e o atendimento no planejamento reprodutivo ${ }^{14}$. Neste mesmo contexto, competências foram identificadas para médicos, enfermeiras e farmacêuticos na APS para subsidiar as ações destes profissionais na prática da SSR ${ }^{15}$ e competências de enfermeiras de saúde pública relacionadas ao planejamento familiar, que contemplam aspectos educativos e de aconselhamento para anticoncepção ${ }^{16}$.

A Organização Mundial de Saúde possui um número considerável de publicações sobre a temática da SSR e apresentou em 2011, um documento sobre as competências essenciais de saúde sexual e reprodutiva que são desejáveis para uso na APS $^{17}$. Este documento discorre sobre as atitudes, tarefas, conhecimentos e habilidades necessários aos profissionais para promover estes cuidados junto à comunidade na perspectiva de direitos humanos.

Durante a revisão de literatura pertinente ao tema no Brasil, observou-se que os objetos de estudo mais recorrentes têm sido o impacto das ações de planejamento familiar sobre os indivíduos, principalmente, jovens e adolescentes e prevenção de gravidez indesejada ${ }^{18,19}$ e a transmissão de doenças sexualmente transmissíveis ${ }^{20}$. Embora tenham sido encontrados estudos que tratassem sobre o tema na APS, uma pesquisa, que relaciona a competência profissional e a assistência em anticoncepção ${ }^{21}$, verificou que há lacunas nesta competência que, associadas à falta de sistematização do trabalho em equipe, geram distorções na qualidade da atenção.

Após discussão de tal problemática, procedeu-se a formulação da questão de pesquisa: quais são as competências transversais necessárias para que as equipes atuem na atenção à saúde sexual e reprodutiva na APS? Neste contexto, este estudo tem como objetivo construir um referencial de competências transversais para atenção em Saúde Sexual e Reprodutiva na Atenção Primária à Saúde a partir da visão de especialistas.

\section{Método}

Para alcançar os objetivos propostos realizou-se um estudo exploratório descritivo de abordagem mista (quali-quantitativo) com o uso da técnica Delphi. A técnica Delphi embasa-se em um método sistematizado de julgamento de informações, útil para obter consensos de especialistas em temas complexos através de validações articuladas em fases ou ciclos ${ }^{22,23}$. Utilizou-se a Técnica Delphi online ${ }^{23}$ que prevê a circulação de questionários estruturados, repetidas vezes, por um grupo de experts na temática estudada, com a realização de feedback estatístico de cada resposta até o alcance do consenso ${ }^{22}$. Para este estudo optou-se pela técnica Delphi a fim de elaborar uma lista de competências transversais para atuação em saúde sexual e reprodutiva na APS.

A Plataforma Lattes foi acessada para seleção de especialistas, sendo utilizado como critérios de busca: o assunto (saúde sexual, saúde reprodutiva, saúde sexual e reprodutiva na Atenção Básica, saúde sexual e reprodutiva na Atenção Primária à Saúde, direitos sexuais, direitos reprodutivos, e direitos sexuais e reprodutivos), na base de dados de doutores de nacionalidade brasileira. Para refinar a pesquisa foi utilizado como filtro: o idioma português, sendo selecionados 41 especialistas. Os (as) especialistas foram escolhidos de acordo com a competência na área, levando-se em consideração a experiência no assunto, bem como idioma e cultura comuns para evitar interpretações distintas pela diferente percepção social. Dentre os participantes selecionados, três relataram através de e-mail que não tinham interesse em participar da pesquisa, pois estavam afastados da temática da saúde sexual e reprodutiva, resultando em uma amostra de 38 especialistas. A estes foi enviado convite, tendo-se obtido retorno de 18 participantes na primeira e na segunda rodada e 17 na terceira, correspondendo a uma taxa de retorno de $47,36 \%$ e $44,73 \%$ respectivamente.

Os dados foram coletados através de três questionários com questões abertas e fechadas. A operacionalização do Delphi se deu por meio da realização de sucessivas rodadas de questionários. O primeiro questionário constava de três questões abertas para que o grupo de especialistas selecionados listassem três competências que 
acreditassem ser necessárias para o trabalho em equipe na atenção a SSR na APS. O instrumento foi submetido a um pré-teste com três docentes que atuam na área com a finalidade de avaliar o constructo do questionário e certificar que as informações podiam ser entendidas com clareza.

Após análise qualitativa das competências resultantes do primeiro questionário, foi enviado um novo questionário, no qual foi atribuída uma Escala Likert com opções de 1 (discordo muito) a 5 (concordo muito) para cada competência, a fim de que os participantes expressassem sua concordância. Aquelas questões que alcançaram consenso foram extraídas e o questionário foi revisado pelo pesquisador e novamente enviado aos (às) participantes com as informações obtidas na primeira rodada. Em seguida, foi enviado um novo questionário, no qual também foi conferida uma Escala Likert, com as competências que não obtiveram consenso na segunda rodada para ser reavaliadas pelos sujeitos do estudo. A coleta de informações foi mediada pelas tecnologias de informação e comunicação e ocorreu através do Google Docs, ambiente virtual disponível na internet que permite o uso de várias mídias, linguagens e recursos de modo organizado, além da elaboração e socialização de informações de modo coletivo ${ }^{24}$.

Na técnica Delphi a análise das informações ocorre concomitante à coleta de dados, sendo empregada uma abordagem de análise quali-quantitativa. A análise qualitativa foi utilizada para a produção dos enunciados das competências que surgiram no primeiro questionário, baseando-se na competência listada e seu comentário $)^{25}$, resultando em 51 sugestões de competências. Foram criados termos-chave com o objetivo de facilitar o agrupamento de componentes comuns. Para a padronização dos enunciados utilizou-se um formato composto por verbo e substantivo, a partir do entendimento de que a competência não pode ser separada da ação".

A construção dos enunciados também levou em consideração recomendações da Organização Mundial da Saúde de que as competências devem ser abrangentes o suficiente para serem utilizadas no âmbito internacional ou nacional, e ao mesmo tempo específicas para subsidiar a tomada de decisões, bem como ser relevantes para a prática ${ }^{26}$.

Da análise e compilação das respostas resultaram 36 competências. Estas foram classificadas em quatro áreas de domínio ${ }^{17}$ : Ética e princípios profissionais; Liderança e gestão; Trabalho com a comunidade, saúde e educação, aconselhamento e avaliação; Provisão do cuidado.
A análise descritiva foi utilizada com o intuito de estabelecer um consenso entre os participantes nas segunda e terceira rodadas do Delphi. O critério adotado foi de 70\% para os valores $4 \mathrm{e}$ 5 da escala de Likert (concordo e concordo muito $)^{22,27}$.

Os preceitos éticos foram seguidos em todas as fases do estudo em consonância com o que preconiza a Resolução no 466/12 do Ministério da Saúde ${ }^{28}$. O projeto de pesquisa foi aprovado pelo Comitê de Ética em Pesquisa (CEP) da Universidade Federal do Rio Grande do Sul.

\section{Resultados e discussão}

A análise das 51 sugestões de competências da primeira rodada resultou em 36 enunciados de competências, que foram classificadas em quatro áreas de domínio. Estas foram consideradas pelos especialistas nas segunda e terceira rodada da Técnica Delphi.

Na segunda rodada do Delphi, conforme evidencia-se na Tabela 1, quatro competências não obtiveram o consenso estabelecido nesse estudo de $70 \%$ nos escores 4 e 5 .

As competências que não obtiveram consenso na segunda rodada foram novamente enviadas aos participantes para apreciação na terceira rodada. Os resultados estão apresentados na Tabela 2.

A partir da análise das competências no domínio ética e princípios profissionais evidencia-se que os especialistas preocupam-se com a atuação profissional pautada na comunicação dialógica, no acolhimento, na empatia, no respeito e desenvolvimento da confiança, na ética, na cultura como determinante de saúde, na resolução de problemas, na igualdade e, no sigilo profissional. É indiscutível que estas competências influenciam na qualidade da atenção prestada nos serviços de atenção primária no âmbito da sexualidade e reprodução.

Em relação à comunicação dialógica, observa-se que escutar e desenvolver o diálogo durante a assistência na SSR depende muito da interação entre profissional de saúde e o usuário. A partir do entendimento da comunicação dialógica, vislumbram-se algumas fragilidades no modelo de comunicação e a necessidade de formação dos trabalhadores de saúde, desde a graduação, com saberes que viabilizem o exercício de práticas comunicativas dialógicas. Além disso, é fundamental proporcionar espaços de formação continuada nos cenários de práticas, de forma a fomentar 
Tabela 1. Médias, desvio padrão e percentual dos escores 4 e 5 das competências, por área de domínio, obtidas na segunda rodada. Porto Alegre, RS, 2016.

\begin{tabular}{|c|c|c|c|}
\hline Domínio/Competências & Média & $\begin{array}{l}\text { Desvio } \\
\text { Padrão }\end{array}$ & $\begin{array}{l}\text { Percentual \% } \\
\text { (escores } 4 \text { e } 5 \text { ) }\end{array}$ \\
\hline \multicolumn{4}{|l|}{ Ética e princípios profissionais } \\
\hline Realiza escuta ativa & 4,61 & 0,50 & 100 \\
\hline Estabelece diálogo a fim de promover o compartilhamento de saberes & 4,61 & 0,58 & 94,44 \\
\hline Comunica-se de forma dialógica & 4,5 & 0,46 & 94,44 \\
\hline Demonstra capacidade de acolhimento livre de preconceitos e julgamentos & 4,72 & 0,46 & 100 \\
\hline Demonstra empatia, respeito e desenvolve a confiança durante os atendimentos prestados & 4,61 & 0,60 & 94,44 \\
\hline $\begin{array}{l}\text { Compreende os fundamentos éticos/bioéticos e da humanização na atenção à saúde } \\
\text { centrados na pessoa e na abordagem familiar }\end{array}$ & 4,77 & 0,42 & 100 \\
\hline Considera o contexto cultural, econômico e social dos indivíduos & 4,61 & 0,69 & 88,88 \\
\hline Respeita os saberes e diferentes culturas & 4,55 & 0,70 & 88,88 \\
\hline Promove o empoderamento dos sujeitos & 3,16 & 1,46 & 50 \\
\hline Demonstra iniciativa para resolução de problemas & 4,38 & 1,03 & 94,44 \\
\hline Reconhece o outro como uma vida que vale o investimento profissional & 4,11 & 0,96 & 72,22 \\
\hline Reconhece suas crenças e valores em relação e valores em relação àquelas dos usuários & 2,94 & 1,30 & 38,88 \\
\hline Assegura o sigilo profissional & 4,83 & 0,38 & 100 \\
\hline \multicolumn{4}{|l|}{ Liderança e gestão } \\
\hline $\begin{array}{l}\text { Garante o acesso a exames de sorologias e medicamentos para as doenças sexualmente } \\
\text { transmissíveis }\end{array}$ & 3 & 1,37 & 33,33 \\
\hline Conhece a rede para possíveis encaminhamentos & 4,38 & 0,63 & 100 \\
\hline Promove a longitunalidade do cuidado & 4,5 & 0,64 & 100 \\
\hline Promove a intersetorialidade & 4,44 & 0,61 & 94,44 \\
\hline \multicolumn{4}{|l|}{ Trabalho com a comunidade, saúde e educação, aconselhamento e avaliação } \\
\hline $\begin{array}{l}\text { Conhece os marcos referenciais políticos e legais, nacionais e internacionais sobre os } \\
\text { direitos na Saúde Sexual e Reprodutiva }\end{array}$ & 4,72 & 0,46 & 100 \\
\hline $\begin{array}{l}\text { Articula conhecimento interdisciplinar inerentes à saúde da população na área de Saúde } \\
\text { Sexual e Reprodutiva }\end{array}$ & 4,6 & 0,50 & 100 \\
\hline Prove aconselhamento e encaminhamentos dos casos de violência sexual & 4,72 & 0,46 & 100 \\
\hline Promove e incentiva o autocuidado na Saúde Sexual e Reprodutiva & 4,61 & 0,50 & 100 \\
\hline $\begin{array}{l}\text { Articula atividades educativas em saúde que abordem a saúde sexual e reprodutiva de } \\
\text { homens, mulheres e jovens }\end{array}$ & 4,72 & 0,46 & 100 \\
\hline Promove a Saúde Sexual e Reprodutiva de indivíduos, famílias e comunidade & 4,55 & 0,70 & 88,88 \\
\hline $\begin{array}{l}\text { Compreende a dinâmica social e cultural considerando aspectos de gênero, classe, raça, } \\
\text { etnia e diversidade social }\end{array}$ & 4,66 & 0,48 & 100 \\
\hline \multicolumn{4}{|l|}{ Provisão do cuidado } \\
\hline Atua junto à mulher, ao homem, à família e à comunidade no âmbito da pré-concepção & 4,72 & 0,46 & 100 \\
\hline $\begin{array}{l}\text { Atua junto à mulher, ao homem, à família e à comunidade no âmbito do Planejamento } \\
\text { reprodutivo }\end{array}$ & 4,72 & 0,46 & 100 \\
\hline Atua junto à mulher, ao homem, à família e à comunidade no âmbito do Pré-natal & 4,77 & 0,42 & 100 \\
\hline Atua junto à mulher, ao homem, à família e à comunidade no âmbito do Parto & 4,38 & 0,97 & 77,77 \\
\hline Atua junto à mulher, à família e à comunidade no âmbito do Climatério & 4,61 & 0,60 & 94,44 \\
\hline Atua junto à mulher, à família e à comunidade no âmbito da menopausa & 4,44 & 0,65 & 88,88 \\
\hline Atua junto ao homem, à família e à comunidade no âmbito da Andropausa & 3,88 & 1,27 & 66,66 \\
\hline $\begin{array}{l}\text { Orienta e administra ações de atenção à mulher e ao homem no contexto da Saúde } \\
\text { Sexual e Reprodutiva }\end{array}$ & 4,55 & 0,51 & 100 \\
\hline Realiza aconselhamento pré e pós testes paras as coletas de exames & 4,77 & 0,42 & 100 \\
\hline Elege os diagnósticos de acordo com sua área profissional & 4,55 & 0,61 & 94,44 \\
\hline $\begin{array}{l}\text { Apresenta capacidade de atuar com resolutividade frente as disfunções sexuais } \\
\text { masculinas e femininas }\end{array}$ & 4,33 & 0,68 & 88,88 \\
\hline Demonstra capacidade técnica para exercer a clínica na Saúde Sexual e Reprodutiva & 4,61 & 0,50 & 100 \\
\hline
\end{tabular}


Tabela 2. Médias, desvio padrão e percentual dos escores 4 e 5 das competências, por área de domínio, obtidas na terceira rodada. Porto Alegre, RS, 2016.

\begin{tabular}{|c|c|c|c|}
\hline $\begin{array}{c}\text { Domínio/ } \\
\text { Competências }\end{array}$ & Média & $\begin{array}{l}\text { Desvio } \\
\text { Padrão }\end{array}$ & $\begin{array}{l}\text { Percentual \% } \\
\text { (escores } 4 \text { e } 5 \text { ) }\end{array}$ \\
\hline \multicolumn{4}{|l|}{$\begin{array}{l}\text { Ética e princípios } \\
\text { profissionais }\end{array}$} \\
\hline $\begin{array}{l}\text { Promove o } \\
\text { empoderamento } \\
\text { dos sujeitos }\end{array}$ & 3,41 & 1,17 & 52,94 \\
\hline $\begin{array}{l}\text { Reconhece suas } \\
\text { crenças e valores em } \\
\text { relação e valores em } \\
\text { relação àquelas dos } \\
\text { usuários }\end{array}$ & 3,70 & 1,10 & 58,82 \\
\hline \multicolumn{4}{|l|}{ Liderança e gestão } \\
\hline $\begin{array}{l}\text { Garante o acesso } \\
\text { a exames de } \\
\text { sorologias e } \\
\text { medicamentos } \\
\text { para as doenças } \\
\text { sexualmente } \\
\text { transmissíveis }\end{array}$ & 3,23 & 1,20 & 41,17 \\
\hline \multicolumn{4}{|l|}{ Provisão do cuidado } \\
\hline $\begin{array}{l}\text { Atua junto ao } \\
\text { homem, família } \\
\text { e comunidade } \\
\text { no âmbito da } \\
\text { andropausa }\end{array}$ & 3,58 & 0,93 & 52,94 \\
\hline
\end{tabular}

Fonte: Dados da pesquisa, Telo SV, Porto Alegre, 2016.

o compartilhamento de conhecimentos que proporcionem à compreensão e o entendimento entre os atores envolvidos no ato comunicativo ${ }^{29,30}$.

As competências que obtiveram consenso neste domínio, exceto a "Reconhece o outro como uma vida que vale o investimento profissional", foram relacionadas como essenciais para atuação em SSR, na APS, na literatura internacional ${ }^{15,17}$. Esta competência foi relacionada pelos especialistas ao princípio da igualdade:

É preciso poder reconhecer que o outro (e sua vida) é importante e vale o investimento do profissional (ESP 4).

No entanto, implementá-la de modo universal e integral ainda é um grande desafio para a APS, tendo em vista a diversidade de ações e as dificuldades de acesso que esbarram em preconceitos e tabus, por parte dos profissionais, que prejudicam o cuidado de pessoas em situação de rua, vivendo em presídios, em situação de prostituição, jovens, idosos e população LGBT. Estas pessoas já têm o seu cuidado tradicionalmente marginalizado, e quando não acessam os serviços se tornam mais vulneráveis a contrair doenças sexualmente transmissíveis, piorar sua condição de saúde em relação a patologias já instaladas, submetendo-se a práticas inseguras e que podem causar sérias complicações ${ }^{1}$. Neste domínio duas competências não obtiveram o consenso. A competência que abordou o empoderamento dos sujeitos obteve resultados controversos, tendo sido considerada muito importante e como sendo difícil de ser promovida. Em relação às crenças e valores, os especialistas relacionaram a competência com a influência de suas próprias crenças e valores no cuidado prestado. Estes resultados indicam a dificuldade dos especialistas para visualizar a necessidade dos profissionais, incentivar as pessoas a buscar seus direitos, fortalecer a autoestima e controlar suas relações pessoais e sociais.

No domínio liderança e gestão, as competências identificadas envolveram aspectos relacionados ao trabalho em rede, preconizando a longitudinalidade do cuidado e a prática de ações intersetoriais que promovam a saúde sexual e reprodutiva. Apesar da longitudinalidade ser um princípio norteador da APS,ela não foi considerada como competência na perspectiva da saúde sexual e reprodutiva em outros estudos.

Para Higa et al. ${ }^{31}$, o Programa Saúde nas Escolas (PSE) é exemplo de política intersetorial na SSR, entre os Ministérios da Saúde e da Educação, organizado em eixos que abordam a avaliação das condições de saúde de alunos, promoção de práticas em saúde e prevenção de doenças, bem como formação de educadores e educação continuada para profissionais da saúde. No programa também é preconizado o monitoramento de ações para melhora da qualidade de vida da comunidade escolar.

A garantia do acesso a exames de sorologias e medicamentos para as doenças sexualmente transmissíveis foi uma competência que não obteve consenso neste domínio. Para a $\mathrm{OMS}^{32}$, o acesso a exames para detecção de doenças sexualmente transmissíveis e a medicamentos essenciais devem ser garantidos pelos serviços de APS como parte do direito à saúde e devem ser ofertados pelos profissionais aos usuários do serviço. Apesar da legislação e da literatura preconizarem a importância do acesso a exames e medicamentos na APS, os sujeitos do estudo relacionaram esta competência como de responsabilidade da gestão. Esta leitura parte de uma abordagem simplista da atuação do profissional de saúde da APS junto a comunidade e desresponsabiliza o mesmo de exigir que a população tenha este acesso 
garantido, implicando a responsabilidade do cuidado somente para a gestão.

Em relação ao domínio trabalho com a comunidade, saúde e educação, aconselhamento e avaliação foram identificadas competências que abordaram os referenciais teóricos, a atuação interdisciplinar, o aconselhamento e o encaminhamento dos casos de violência sexual, a promoção do autocuidado, o trabalho educativo, a promoção do cuidado e, a compreensão da dinâmica social e cultural considerando aspectos de gênero, classe, raça, etnia e diversidade social.

A promoção do autocuidado não foi identificada nos textos utilizados como referência para este estudo, embora seja uma recomendação para abordagem de saúde pública em SSR ${ }^{32}$.

$\mathrm{O}$ trabalho interdisciplinar, como fundamento básico da APS foi abordado na competência "Articula conhecimento interdisciplinar inerente à saúde da população". Para esta competência os especialistas referiram a importância da contribuição de cada disciplina:

$O$ objeto profissional se define com o agir da profissão e para isto se faz necessário diferentes habilidades adquiridas durante a formação acadêmica do enfermeiro (ESP 7).

$\mathrm{O}$ trabalho interdisciplinar e em equipe é o eixo norteador do documento da $\mathrm{OMS}^{17}$, que relaciona as competências essenciais para a assistência na saúde sexual e reprodutiva na APS. Para Cappielo et al..$^{15}$ colaborar com os membros da equipe interdisciplinar através de objetivos comuns, comunicação eficaz, respeito mútuo e compreensão dos papéis de cada um é imprescindível na prestação de cuidados de saúde sexual e reprodutiva.

O enfrentamento da epidemia da AIDS constitui um exemplo da necessidade do uso da interdisciplinaridade, já que é necessária a compreensão das atitudes e comportamentos, estilos de vida e os aspectos socioculturais envolvidos na exposição ao risco. O cuidado prestado por diversos profissionais, que trabalham no atendimento a pessoas com doenças sexualmente transmissíveis ou HIV, precisa refletir a subjetividade que envolve viver com essas doenças, investir em novas ações e construir conhecimentos para a obtenção de bons resultados de promoção da saúde ${ }^{33}$.

Os especialistas também reconheceram a APS como alternativa para o enfrentamento da violência. Prover aconselhamento e encaminhamento dos casos de violência sexual é a competência mais desafiadora para a APS neste domínio tendo em vista a complexidade envolvida, a proximidade de relações e vínculos, a necessidade de rápida identificação e a dificuldade de modificar a situação $0^{34}$. Este é um problema de grande magnitude e competências transversais podem contribuir para reorganizar as práticas assistenciais, de capacitação dos profissionais de saúde e da realização de parcerias com instituições públicas e privadas, bem como a organização de grupos de apoio para as mulheres e as famílias.

No domínio provisão do cuidado foram relacionadas competências para a atuação no planejamento reprodutivo, na pré-concepção, no pré-natal, na assistência ao parto e cuidado no climatério, bem como a administração de ações, a realização de aconselhamento, a escolha de diagnósticos, a resolutividade frente às disfunções sexuais e a capacidade técnica para o cuidado clínico. Tais competências foram consideradas fundamentais para a qualificação dos cuidados prestados na saúde sexual e reprodutiva no âmbito da $\mathrm{APS}^{15-17}$, e como competências necessárias para a atuação profissional na atenção à saúde sexual e reprodutiva ${ }^{1}$.

A competência que relacionou o cuidado na andropausa não obteve consenso entre os especialistas. Os sujeitos referiram não ter conhecimento para discutir sobre esta competência, mesmo sendo pesquisadores da temática da saúde sexual e reprodutiva na APS. Apesar do incentivo em incluir homens no cuidado na saúde sexual e reprodutiva, através de políticas e ações, este resultado sinaliza para uma fragilidade no que tange à construção e divulgação do conhecimento necessário à atuação na saúde do homem ${ }^{35}$.

Segundo Separavich e Canesqui ${ }^{36}$, conceitos como disfunção erétil, andropausa e reposição hormonal masculina são bastante abordados ultimamente na mídia e nos espaços da agenda pública e política da saúde masculina, e necessitam ser priorizados também na assistência prestada no âmbito da APS. Schraiber et al. ${ }^{37}$ destacam que a maioria dos atendimentos da APS envolvem problemas de saúde das mulheres, uma vez que a prevenção e o cuidado em saúde são socialmente considerados como tarefas essencialmente femininas. Desta forma, a mesma lógica estrutura os serviços, intervenções e interlocuções entre profissionais de saúde e usuários, reproduzindo relações tradicionais de gênero.

Competências importantes identificadas na literatura internacional abordando a assistência no período pós-parto, nos casos de aborto (sejam eles provocados ou não) e pertinentes a infertilidade não foram relacionadas neste estudo. Também não foram abordadas competências envolvendo a saúde mental e o uso de drogas. 
Apesar da APS ser referência para realização de aconselhamento e testagem rápida para HIV e outras doenças, a assistência e o tratamento de doenças sexualmente transmissíveis também não foram identificadas pelos especialistas como uma competência necessária para a atuação na SSR.

Embora a saúde da mulher e do homem tenham sido abordados em várias competências, as estratégias de rastreamento para câncer de mama, útero e cânceres masculinos, preconizadas pelo Ministério da Saúde, não foram descritas como uma habilidade específica. Este fato indica a necessidade da ampliação do enfoque para além das ações preventivas e campanhistas nas questões de SSR na perspectiva do cuidado integral.
Este referencial de competências confirma a necessidade de ampliação do leque de atuação dos profissionais da APS para novas frentes de cuidado contemplando de modo universal e equânime a todos os indivíduos, constituindo instrumento para o desenvolvimento de ações que contemplem a SSR, incluindo populações tradicionalmente marginalizadas. As competências refletem o estágio atual de discussão e consolidação de conhecimentos na realidade brasileira e constituem elemento para interlocução internacional na discussão a respeito da organização da assistência em SSR. Poderão subsidiar a formação de profissionais de saúde e, por serem transversais o planejamento de ações e as práticas das equipes no âmbito da saúde Sexual e Reprodutiva.

\section{Colaboradores}

SV Telo e RR Witt tiveram igual participação em todas as etapas de elaboração do artigo. 


\section{Referências}

1. Brasil. Ministério da Saúde (MS). Saúde sexual e saúde reprodutiva. Brasília: MS; 2013.

2. Lemos A. Direitos sexuais e reprodutivos: percepção dos profissionais da atenção primária à saúde. Saúde Debate 2014; 38(101):244-253.

3. Souza KV, Tyrrell MAR. Os fatos \& atos relacionados ao (difícil) exercício dos direitos sexuais e reprodutivos: em recortes, o processo de viver de um grupo de mulheres de classes populares. Texto Contexto - Enfermagem 2007; 16(1):47-54.

4. Brasil. Ministério da Saúde (MS). Política nacional de atenção integral à saúde da mulher. Brasília: MS; 2011.

5. Ramos FIS. Análise histórica das politicas de planejamento familiar no Brasil [tese]. Rio de Janeiro: Universidade Estadual do Rio de Janeiro; 2008.

6. Corrêa S, Ávila MB. Direitos sexuais e reprodutivos: pauta global e percursos brasileiros. In: Berquó E, organizador. Sexo e vida: panorama da saúde reprodutiva no Brasil. Campinas: Unicamp; 2003. p.17-78.

7. Rios RR. Para um direito democrático da sexualidade. Horizontes Antropológicos 2006; 12(26):71-100.

8. Poli MEH. A anticoncepção como instrumento do planejamento familiar e da saúde. Scimed 2006; 16(4):168171.

9. Ramos MN. A pedagogía das competências: autonomía ou adaptação? 4a ed. São Paulo: Cortez; 2011.

10. Gebbie MK, Gill ES. Competency-to-curriculum toolkit. New York: Association for Prevention Teaching and Research, 2008. [acessado 2016 Jun 25]. Disponível em: http://www.phf.org/resourcestools/Documents/Competency_to_Curriculum_Toolkit08.pdf

11. Hutchinson J1, Evans D, Sutcliffe LJ, MacQueen RA, Davies J, Estcourt CS. Competency: development and evaluation of a new clinical training and assessment programme in sexual health for primary care health professionals. Int. J. STD AIDS 2012; 23(8):589-592.

12. Shawe J, Cox S, Penny N, White A, Wilkinson C. A service-based approach to nurse training in sexual and reproductive health care. J Fam Plann Reprod Health Care 2013; 39(4):285-288.

13. Khanam M, Perveen S, Mirza S. Reproductive and sexual health issues: knowledge, opinion and attitude of medical graduates from Karachi. J Pak Med. Assoc. 2011; 61(7):648-652.

14. Akers AY, Gold MA, Borrero S, Santucci A, Schwarz EB. Providers' perspectives on challenges to contraceptive counseling in primary care. J Women Health 2010; 19(6):1163-1170.

15. Cappielo J, Levi A, Nothnagle M. Core competencies in sexual and reprodutive health for theinter professional primary care team. Contraception 2016; 93(5):483-545.

16. Hewitt CM, Roye C, Gebbie KM. Core competency model for the family planning public health nurse. Public Health Nursing 2014; 31(5):472-479.
17. World Health Organization (WHO). Sexual and reproductive health core competencies in primary care: attitudes, knowledge, ethics, human rights, leadership, management, teamwork, community work, education, counselling, clinical settings, service, provision. Geneva: WHO; 2011. Disponível em: http://apps.who.int/iris/ bitstream/10665/44507/1/9789241501002_eng.pdf > . [acessado 15 set. 2016.

18. Dirksen RR1, Shulman B, Teal SB, Huebschmann AG. Contraceptive counseling by general internal medicine faculty and residents. J Women Health 2014; 23(8):707713.

19. Ribeiro CPS, Martins MC, Gubert FA, Almeida NMGS, Silva DMA, Afonso LR. Percepção de adolescentes escolares sobre transformações corporais, gravidez e caderneta de saúde do adolescente. Revista Cubana de Enfermería 2016; 32(1):27-36.

20. Santos JCA. O enfermeiro e o ensino na assistência às doenças sexualmente transmissíveis: revisão integrativa. Revista Brasileira de Educação e Saúde 2016; 6(2):812.

21. Moura ERF, Silva RM. Competência profissional e assistência em anticoncepção. Rev Saude Publica 2005; 39(5):795-801.

22. Castro AV, Rezende M. A Técnica Delphi e seu uso na pesquisa de enfermagem: revisão bibliográfica. Rev. Min. Enferm. 2009; 13(3):429-434.

23. Silva GF, Moura MAV, Almeida MVS, Sá SPC, Queiroz ABA. Influências do climatério para o envelhecimento na percepção de mulheres idosas: subsídios para a enfermagem. Rev. Eletr. Enf. 2016; 17(3). [acessado 2016 Jun 23]. Disponível em: https://revistas.ufg.br/fen/article/view/29072/20750

24. Scarparo AF, Laus AM, Azevedo ALCS, Freitas MRI, Gabriel CS, Chaves LDP. Reflexões sobre o uso da técnica Delphi na pesquisa em enfermagem. Revista da Rede de Enfermagem do Nordeste 2012; 13(1):242-251.

25. Souza PA, Frade MHLBC, Mendonça DMMV. Um modelo de organização e partilha de informações de enfermagem entre hospital e centro de saúde: estudo Delphi. Acta Paulista de Enfermagem 2005; 18(4):368-381.

26. World Health Organization (WHO). Competency in nursing. Geneva: WHO; 2003.

27. Witt RR, Almeida MCP. Identification of nurses' competencies in primary health care through a Delphi study in Southern Brazil. Public Health Nursing 2008; 25(4):336-343.

28. Brasil. Ministério da Saúde (MS). Conselho Nacional de Saúde. Resolução no 466, de 12 de dezembro de 2012. Diário Oficial da União 2013; 13 dez.

29. Raimundo J, Cadete MMM. Escuta qualificada e gestão social entre os profissionais de saúde. Acta Paul. Enferm. 2012; 25(n. esp. 2):61-67.

30. Teixeira RR. Humanização e atenção primária à saúde. Cien Saude Colet 2005; 10(3):585-597. 
31. Higa EFR, Bertolin FH, Maringolo LF, Ribeiro TFSA, Ferreira LHK, Oliveira VASC. A intersetorialidade como estratégia para promoção da saúde sexual e reprodutiva dos adolescentes. Interface (Botucatu) 2015; 19:879-891.

32. World Health Organization (WHO). Sexual health, human rights and the law. Geneva: WHO; 2015. [acessado 2016 Set 15]. Disponível em: http://apps.who.int/iris/ bitstream/10665/175556/1/9789241564984_eng.pdf

33. Araújo IA, Queiroz ABA, Moura MAV, Penna LHG. Representações sociais da vida sexual de mulheres no climatério atendidas em serviços públicos de saúde. Texto e Contexto 2013; 22(1):114-122.

34. Soares JSF, Lopes MJM, Njaine K. Violência nos relacionamentos afetivosexuais entre adolescentes de Porto Alegre, Rio Grande do Sul, Brasil: busca de ajuda e rede de apoio. Cad Saude Publica 2013; 29(6):1121-1130.

35. Brasil. Ministério da Saúde (MS). Política nacional de atenção integral à saúde do homem: princípios e diretrizes. Brasília: MS; 2009.

36. Separavich MA, Canesqui AM. Saúde do homem e masculinidades na política nacional de atenção integral à saúde do homem: uma revisão bibliográfica. Saúde Soc 2013; 22(2):415-428.

37. Schraiber LB, Figueiredo WS, Gomes R, Couto MT, Pinheiro TF, Machin R, Silva GSN, Valença O. Necessidades de saúde e masculinidades: atenção primária no cuidado aos homens. Cad Saude Publica 2010; 26(5):961-970.

Artigo apresentado em 27/09/2016

Aprovado em 06/11/2016

Versão final apresentada em 08/11/2016 